é mostrar a conexão desta emergente disciplina, com questões da própria regulação das nanotecnologias.

O desenvolvimento da ciência e tecnologia depende fundamentalmente de métodos, ferramentas e técnicas. As nanotecnologias têm entre suas techniques de choix as microscopias, aqui tomadas em senso largo. $\mathrm{O}$ artigo de Fernando Galembeck, atual diretor do Laboratório Nacional de Nanotecnologia, no Centro Nacional de Pesquisas em Energia e Materiais, trata desse assunto, enfoca as microscopias de sonda e, mais especificamente, a microscopia de força atômica (AFM) e toda uma gama de configuraçôes que a transformaram numa verdadeira "plataforma de microscopias". São apresentados vários exemplos onde essas ferramentas podem ser utilizadas com sucesso e que vão desde a avaliação de aspectos topográficos até a medida de potenciais elétricos de nanomateriais.

Finalmente, Mahendra Rai, professor da Universidade SGB Amravati, na Índia, tratará daquilo que vem sendo colocado como uma alternativa bastante interessante para a produção de alguns tipos de nanomateriais (nanopartículas), ou seja: a nanotecnologia verde (green nanotechnology). No caso em questão, para a produção dos nanomateriais de interesse e com funcionalidades definidas são usados extratos de plantas, biomassa, fungos, bactérias, entre outros. Os nanomateriais formados podem ser empregados como agentes antibacterianos, antimaláricos, no tratamento de água para o consumo humano etc, podendo vir a impactar políticas públicas de saúde em países menos desenvolvidos.

Muitos artigos reportados na literatura internacional colocam as nanotecnologias como verdadeira panaceia, ou seja: com capacidade para resolver todos os problemas. De nosso ponto de vista, vemos as nanotecnologias como uma grande plataforma de conhecimento científico-tecnológico, intrinsecamente inovadora e multidisciplinar, que aportará o desenvolvimento de novos produtos e soluções tecnológicas relevantes, mas que, no entanto, em muitos casos, terá que se mostrar mais eficiente, mais segura e apresentar uma relação custo/benefício favorável, quando comparadas com as tecnologias convencionais, estabelecidas e testadas anos a fio. Acreditamos, ainda, que ocorrerão situações nas quais teremos sistemas híbridos, constituídos por tecnologia convencional e nanotecnológica, atuando sinergisticamente.

Em vista dos pontos aqui levantados, não nos parece um exagero desmesurado fazermos coro àqueles que colocam as nanotecnologias como uma nova revolução científica e tecnológica.

Oswaldo Luiz Alves é professor titular e coordenador científico do Laboratório de Química do Estado Sólido (LQES), do Instituto de Quimica da Unicamp. Membro da Academia Brasileira de Ciências $(A B C)$. Email: oalves@iqm.unicamp.br.

\section{NOTA BIBLIOGRÁFICA}

1. Para conhecer aspectos básicos da nanotecnologia veja Alves, O.L., "Cartilha sobre nanotecnologia", Agência Brasileira de Desenvolvimento Industrial (ABDI). 2010. Disponível em PDF: . http://Iqes.iqm. unicamp.br/images/publicacoes_teses_livros_resumo_cartilha_ abdi.pdf (Acesso em maio de 2013).

\section{CONSIDERACÕES SOBRE o programa brasilemo DE NANOTEGNOLOGIA}

\author{
Flávio Plentz \\ Adalberto Fazzio
}

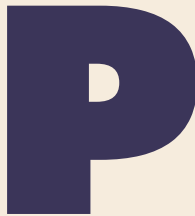

assaram-se cerca de 25 anos desde que a nanociência e nanotecnologia $(\mathrm{N} \& \mathrm{~N})$ começaram a integrar de forma mais consistente a agenda da comunidade científica e tecnológica. Hoje as N\&N permeiam essencialmente todos os setores da sociedade e geram opiniōes, por vezes, contraditórias. As reaçōes às nanotecnologias vão, atualmente, do mais contagiante otimismo, pelas possibilidades que oferecem para fazer frente a muitos dos grandes desafios da humanidade, como nas áreas da energia, saúde, agricultura e pecuária, processamento e armazenamento de informação, para citar apenas alguns, até o temor por possíveis impactos ambientais e efeitos adversos para a saúde humana e animal.

O lançamento da chamada "Iniciativa Americana de Nanotecnologia" é tomada por muitos como sendo um marco nas açōes governamentais articuladas, orgânicas, direcionadas especificamente para alavancar, incentivar, fomentar, coordenar e gerir as políticas e açôes em N\&N. Hoje, todos os países ditos desenvolvidos e um número grande e crescente de países emergentes e em desenvolvimento têm iniciativas e programas nacionais em $\mathrm{N} \& \mathrm{~N}$, inclusive o Brasil.

A revista Ciência \& Cultura já publicou alguns artigos abordando o tema da $N \& N(1 ; 2)$, o primeiro deles apontando para a necessidade de uma iniciativa brasileira de nanotecnologia que permitisse ao Brasil ser parte atuante no cenário mundial e o segundo apresentando algumas das fantásticas possibilidades que a nanotecnologia traz em termos de avanços, especialmente na área de saúde. Neste artigo o que pretendemos é apresentar o desenho do atual Programa Brasileiro de Nanotecnologia (PBN) que tem como bases: (i) a integração de diversos ministérios e agências; (ii) a melhoria da gestão e do acompanhamento dos programas, ações e iniciativas; (iii) a consolidação de uma infraestrutura moderna e, em especial, aberta e acessível, para a pesquisa, desenvolvimento e inovação (P,D\&I); (iv) prover meios, instrumentos, e ambiente regulatório adequado para que as nanotecnologias possam ser escalonadas, industrializadas e comercializadas e, por fim, mas com destaque para sua importância; (v) a formação e fixação de recursos humanos, sem os quais quaisquer esforços ou açōes ficam comprometidos.

No Ministério da Ciência, Tecnologia e Inovação (MCTI) a N\&N está sob a responsabilidade da Secretaria de Desenvolvimento Tecnológico e Inovação (Setec) através da Coordenação Geral de Micro e Nanotecnologias (CGNT). A CGNT conta com o suporte do Comitê Consultivo de Nanotecnologia (CCNANO) composto por pesquisadores, membros de entidades representativas do setor privado e pessoas ligadas a órgãos, agências, empresas e instituições do governo federal. 
HISTÓRICO Apesar dos grandes avanços nas pesquisas científicas brasileiras, a produção e/ ou comercialização de produtos com nanotecnologia nacional ainda são pouco expressivas quando comparadas a países cujas indústrias apresentam elevado grau de desenvolvimento. Embora, a partir principalmente do início da década de 1990, muitos cientistas já trabalhassem nessa área, essa tecnologia passou a ter repercussão mundial, como comentamos anteriormente, a partir de 2001, com o lançamento do programa americano "National Nanotecnology Initiative - NNI". Na mesma época a China lançou seu programa, criando o Comitê Nacional para Nanociência e Nanotecnologia (NSCNN) e publicando o programa "National Nanotechnology Development Strategy 2001-2010”, semelhante ao NNI, apesar de o país já financiar ações específicas de N\&N desde o início da década de 1990.

No Brasil, em 2001, o Conselho Nacional de Desenvolvimento Científico e Tecnológico (CNPq) deu o primeiro passo para o desenvolvimento da nanotecnologia através do apoio à formação de quatro redes cooperativas de pesquisa e quatro Institutos (virtuais) do Milênio, com um investimento de $\mathrm{R} \$ 30$ milhōes, para quatro anos. Dentro dessa iniciativa foi contemplada a primeira proposta que, explicitamente, referia-se ao tema; o "Instituto de Nanociências", que teve a participação de 14 instituiçôes: CBPF; Cetec/MG; CNEN; Funrei/MG; ITP/SE; LNLS; PUC/RJ; UERJ; UFBA; UFF; UFJF; UFMG; UFRJ e UFV.

Em 2004, o MCT lançou, no âmbito do Plano Plurianual (PPA) 2004-2007, o programa "Desenvolvimento da Nanociência e Nanotecnologia"(3). Este programa foi elaborado com base nas recomendações de um grupo de trabalho instituído via Portaria MCT no 252 , de 16.05.2003. Em 2005, o presidente da República, Luiz Inácio Lula da Silva, e o ministro da Ciência e Tecnologia, Sergio Rezende, lançaram um Programa Nacional de Nanotecnologia. Além dos recursos do PPA, esse programa contou com recursos dos fundos setoriais o que elevou o patamar de investimentos do MCT em nanotecnologia e possibilitou ampliar o número de iniciativas nesse tema.

Uma iniciativa que contribuiu decisivamente para uma maior consistência ao complexo brasileiro de nanotecnologia foi a criação do programa "Institutos Nacionais de Ciência e Tecnologia (INCT)”, pela Portaria MCT No 429, de 17 de julho de 2008, que passou a ocupar uma posição estratégica no Sistema Nacional de Ciência, Tecnologia e Inovação (C,T\&I) sendo que 16 INCTs realizam hoje pesquisas diretamente associadas a $\mathrm{N} \& \mathrm{~N}$ nas mais diversas áreas do conhecimento.

No que tange à articulação com a Política de Desenvolvimento Produtivo (PDP), foi realizado um esforço conjunto entre o MCT, Ministério do Desenvolvimento, Indústria e Comércio Exterior (MDIC) e a Associação Brasileira de Desenvolvimento Industrial (ABDI) para a divulgação da nanotecnologia no setor privado, ressaltando a sua importância estratégica como ferramenta à ino- vação. Foram realizados 13 eventos com essa finalidade entre 2008 e 2009.

A formação de redes cooperativas de $\mathrm{P} \& \mathrm{D}$ em N\&N, envolvendo diferentes atores do sistema de inovação, facilitou a transferência de conhecimento e contribuiu para o crescimento e competitividade da indústria nacional. As primeiras redes cooperativas apoiadas pelo MCTI foram as Redes Brasil Nano (Portaria $n^{\circ} 614$, de $1^{\circ}$ de dezembro de 2004).

Em 2010, dezessete novas redes cooperativas foram apoiadas através do edital MCT/CNPq no 74/2010. Em 2011, foi aberta uma chamada pública MCTI/CNPq no 17/2011, "Apoio à criação de redes cooperativas de pesquisa e desenvolvimento em nanotoxicologia e nanoinstrumentação". Todas as redes visavam a solução de gargalos tecnológicos da indústria brasileira. Foram aprovadas seis redes de nanotoxicologia e duas redes de nanoinstrumentação. No total são 25 redes cooperativas apoiadas atualmente pelo MCTI, Conselho Nacional de Desenvolvimento Científico e Tecnológico (CNPq) e pela Financiadora de Estudos e Projetos (Finep) .

Em 2012 a CGNT lançou um edital em conjunto com o CNPq, edital MCTI/CNPq no 16/2012 "Tecnologias inovadoras na produção, prototipagem e/ou aumento de escala em nanotecnologia que apoiou 25 projetos com um investimento total de pouco mais de R \$6 milhões. Esse edital teve uma característica que foi separar metade dos recursos para jovens pesquisadores, contemplados com 15 projetos.

Em 2009, conforme recomendação do comitê executivo da PDP, foi criado o Fórum de Competitividade em Nanotecnologia (FCN) (5). O FCN surgiu como ferramenta estratégica para apoiar a discussão e encaminhamento de iniciativas e programas segundo as dimensões da PDP (6).

A CGNT, em parceria com o Ministério do Desenvolvimento, Indústria e Comércio Exterior (MDIC) e a Associação Brasileira de Desenvolvimento Industrial (ABDI), realizou em 2011 o "10 Workshop Nanotecnologias: da ciência ao mundo dos negócios" (7), na sede do Senai Mario Amato, em São Bernardo do Campo (SP) e em novembro de 2012 foi realizado o "2o Workshop Nanotecnologias: da ciência ao mundo dos negócios" (8), em Fortaleza (CE). Foram apresentados e discutidos casos de sucesso em nanotecnologia de pequenas, médias e grandes empresas nos setores metalomecânico, saúde e alimentos. Os mais de 120 participantes presentes ainda puderam conhecer as ações de nanotecnologia do MCTI, os instrumentos de apoio à inovação do Banco Brasileiro de Desenvolvimento Econômico e Social (BNDES) e da Finep e as oportunidades de parcerias com os INCTs.

Cabe aqui fazer uma menção ao apoio que o MCTI tem dado à questão da nanotoxicologia. O objetivo é capacitar o Brasil para se empenhar no processo de avaliação de riscos e realizar a regulação do uso e aplicação e nanotecnologias. Há intensa atividade nessa área como 
parte do esforço recente para regular o uso de nanotecnologias gerando publicações científicas e também com normas específicas. Em janeiro de 2012, uma edição inteira da revista Environmental Toxicology and Chemistry foi dedicada à questão de nanomateriais e meio ambiente (9) e alguns estudos olham de forma específica para a busca de métodos de mitigação de impactos ambientais de nanomateriais (10).

Certamente os trabalhadores mais expostos hoje a eventuais prejuízos de nanomateriais à saúde humana são os pesquisadores e técnicos envolvidos em Pesquisa, Desenvolvimento e Inovação (PD\&I). Sendo assim, um bom exemplo de documento que busca orientar quanto aos cuidados ao se lidar com nanotecnologias é o manual "Práticas gerais de segurança para trabalhar com nanomateriais criados por engenharia em laboratórios de pesquisa" (General safe practices for working with engineered nanomaterials in research laboratories) (11), publicado pelo Instituto Nacional para Segurança Ocupacional e Saúde dos Estados Unidos da América (National Institute for Occupational Safety and Health - NIOSH).

O Ministério da Educação (MEC) e a Coordenação de Aperfeiçoamento de Pessoal de Nível Superior (Capes) têm ações importantes em nanotecnologia que vão além dos seus papéis já consagrados na criação e consolidação de universidades públicas e programas de pós-graduação. Um exemplo é o acordo de cooperação envolvendo Portugal e Espanha, traduzido, por exemplo, no apoio para projetos de pesquisa utilizarem infraestruturas de pesquisa no estado da arte, em particular o Laboratório Ibérico de Nanotecnologia em Braga, Portugal. A cooperação envolvendo Portugal e Espanha traduziu-se em um edital da Capes lançado no primeiro semestre de 2013 (12).

O PBN tem o objetivo de ser uma base sólida para transformar o Brasil em país científico, tecnológico e economicamente competitivo em nível mundial no que diz respeito à geração e utilização da nanotecnologia como um dos principais motores do desenvolvimento econômico e social. São partes essenciais dessa iniciativa a criação do Comitê Interministerial de Nanotecnologia (CIN) e do Sistema Nacional de Laboratórios em Nanotecnologias (SisNANO), que passamos a descrever a seguir.

GOVERNANÇA O MCTI tomou a iniciativa de instituir o CIN (Portaria Interministerial no 510 , de 09.07.2012) que tem por finalidade integrar a coordenação e a gestão da $\mathrm{PBN}$, contribuindo para o aprimoramento constante e implementação de suas políticas, diretrizes e açôes. Dentro da estrutura da PBN é o CIN, em última instância, o responsável por estabelecer as grandes metas, estratégias e visão de longo prazo para o desenvolvimento de todos os aspectos da nanotecnologia no Brasil, bem como a relação global do Brasil no que diz respeito à nanotecnologia.

Atualmente compõem o CIN: MCTI, Ministério do Meio Ambiente (MMA), Ministério da Agricultura, Pecuária e Abastecimento (Mapa), Ministério da Defesa (MD), MDIC, MEC, Ministério de Minas e Energia (MME), Ministério da Saúde (MS), Ministério do Trabalho e Emprego (MTE) e Ministério das Relações Exteriores (MRE). Participam como convidados, por terem papel central na definição e implementação da PBN, a Agência
Figura 1 - Estrutura de governança do atual Programa Brasileiro de Nanotecnologia

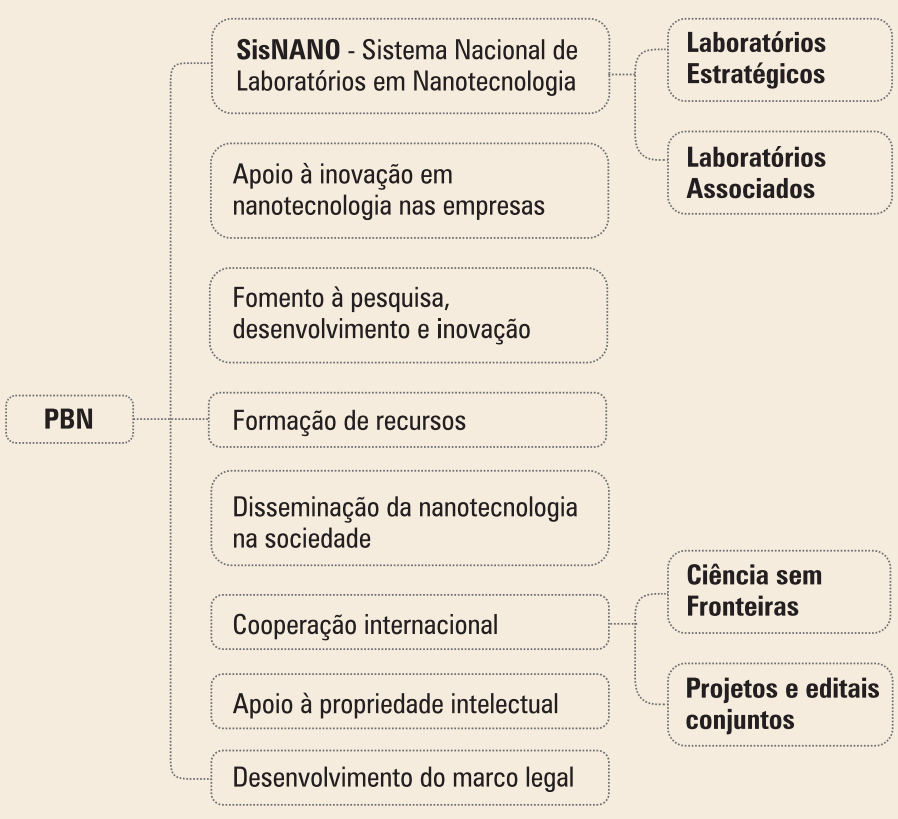

Brasileira de Desenvolvimento Industrial (ABDI), a Agência Nacional de Vigilância Sanitária (Anvisa), o BNDES, o CNPq, o Centro de Gestão e Estudos Estratégicos (CGEE), a Confederação Nacional da Indústria (CNI) e a Finep.

O CIN tem também sob sua responsabilidade: (i) criar e propor mecanismos de fomento e financiamento; (ii) implantar instrumentos de gestão como indicadores, elaboração de séries históricas e estudos de prospecção de cenários e tendências os quais passarão a ter maior consistência, abrangência e, principalmente, maior transparência e visibilidade pública; (iii) realizar o acompanhamento e avaliação dos programas de nanotecnologia, fortalecendo iniciativas de sucesso, reformando, redirecionando ou encerrando iniciativas com resultados insatisfatórios e propondo novas estratégias e ações consoantes com novos horizontes e tendências da "nano". Na figura 1 apresentamos a estrutura de governança da PBN.

SisNANO O Sistema Nacional de Laboratórios em Nanotecnologias (SisNANO) é um sistema formado por um conjunto de laboratórios dedicados à PD\&I, abarcando um amplo espectro de nanotecnologias. Foi instituído pela Portaria no 245 de 5 de abril de 2012 e se apresenta como um sistema de fomento, suporte e gestão de infraestrutura e recursos humanos dedicados à nanotecnologia. A Instrução Normativa no $^{\circ}$, de 15 de junho de 2012, dispõe sobre o regulamento técnico para integração ao Sistema Nacional de Laboratórios em Nanotecnologia e dá outras providências.

Seu objetivo é ampliar o acesso aos laboratórios que fazem parte do SisNANO e, especialmente, institucionalizar e formalizar o com- 
promisso desses laboratórios em atuarem como laboratórios abertos a usuários externos oriundos dos setores público e privado, permitindo maior interação entre pesquisadores e empresas e maiores facilidades para utilização, não apenas da infraestrutura dos laboratórios, mas também de recursos humanos aptos a prestarem assistência no desenvolvimento de projetos de PD\&I ou realização de testes e análises em nanotecnologia.

Em junho de 2012 foi realizada uma chamada pública, amplamente divulgada, para submissão de propostas de laboratórios para compor o SisNANO. Das 50 propostas 26 foram aprovadas depois de análise pelo CCNANO e pela CGNT.

Um ponto importante foi a exigência de que cada proposta fosse encaminhada pelo dirigente máximo da instituição à qual o laboratório estivesse associado. O SisNANO não foi concebido como um sistema de apoio a projetos, grupos de pesquisa ou pesquisadores específicos, mesmo que de alta qualificação, e foi direcionado para laboratórios de caráter claramente multiusuário, que tivessem interface e superposição significativa com projetos, grupos de pesquisa, pesquisadores e empresas, que já tivessem um grau significativo de maturidade e consolidação. Os laboratórios do SisNANO devem assumir várias responsabilidades que exigem um comprometimento institucional. Esses laboratórios saem da esfera pessoal, local ou mesmo regional e assumem um papel nacional de suporte ao desenvolvimento da nanotecnologia.

Pode-se pensar o SisNANO como um instrumento que, a partir de infraestruturas e competências já consolidadas por anos de investimento através de inúmeras açōes e programas dos governos federal, estaduais e municipais cria um conjunto coeso de laboratórios de caráter nacional sob a gestão do MCTI e do CIN. Há duas categorias de laboratórios no SisNANO. Os chamados Laboratórios Estratégi$\cos (\mathrm{LE})$ estão em unidades de pesquisa, institutos ou organizações sociais diretamente ligadas ao governo federal. Esses laboratórios assumem o compromisso de disponibilizarem pelo menos $50 \%$ do tempo para projetos e usuários externos, sejam eles do setor público ou privado. Os LE são os seguintes:

\section{LABORATÓRIOS ESTRATÉGICOS DO SISNANO:}

1. Laboratório de Nanotecnologia para o Agronegócio - LNNA (Embrapa Instrumentação, SP)

2. Centro de Caracterização em Nanotecnologia para Materiais e Catálise - Cenano (INT, RJ)

3. Laboratório Nacional de Nanotecnologia - LNNANO (Cnpem/ SP)

4. Laboratório Multiusuário de Nanotecnologia do Cetene - LMNano (Cetene, PE)

5. Laboratório de Química de Nanoestruturas de Carbono - LQN (CDTN/Cnen, MG)

6. Laboratório Estratégico de Nanometrologia do Inmetro (Inmetro, RJ)

7. Laboratório Multiusuário de Nanociências e Nanotecnologia Labnano (CBPF, RJ)

8. Laboratório Integrado de Nanotecnologia - LIN-Ipen (Ipen/ Cnen, SP)
Os Laboratórios Associados (LA) estão em Instituiçōes de Ciência e Tecnologia (ICTs) como, por exemplo, universidades públicas ou privadas que tenham esse caráter. Os LA assumem os mesmos compromissos de abertura tendo que disponibilizar, no mínimo, $15 \%$ do tempo disponível para usuários externos, nos mesmos moldes dos LE. Os LA são os seguintes:

\section{LABORATÓRIOS ASSOCIADOS DO SISNANO:}

1. Laboratório Regional de Nanotecnologia - LRNANO (UFRGS/RS)

2. Centro de Caracterização e Desenvolvimento de Protocolos para Nanotecnologia - CCDPN (Unesp/SP)

3. Central Analítica em Técnicas de Microscopia (eletrônica e ótica) da Universidade Federal do Ceará (UFC/CE)

4. Laboratório de Síntese de Nanoestruturas e Interação com Biossistemas - Nanobioss (Unicamp/SP)

5. Laboratório de Caracterização Estrutural - LCE (UFSCar/SP)

6. Laboratório Associado de Desenvolvimento e Caracterização de Nanodispositivos e Nanomateriais - LANano (UFMG/MG)

7. Laboratório de Nanobiotecnologia para Desenvolvimento, Prototipagem e Validação de Produtos para o SUS (IBMP/PR)

8. Laboratórios Associados em Nanotecnologia - LARnano (UFPE/PE)

9. Laboratório Associado da UFV (UFV/MG)

10. Laboratório de Nanociência e Nanotecnologia da Amazônia Labnano-Amazon (UFPA/PA)

11. Laboratório de Eletroquímica e Materiais Nanoestruturados LEMN (UFABC/SP)

12. Laboratório de Engenharia de Superfícies e Materiais Nanoestruturados da Coppe - LabEngNano/Coppe (UFRJ/RJ)

13. Laboratório Interdisciplinar para o Desenvolvimento de Nanoestruturas - Linden (UFSC/SC)

14. Núcleo de Bionanomanufatura (IPT/SP)

15. Centro de Componentes Semicondutores - CCS (Unicamp/SP)

16. Núcleo de Apoio à Pesquisa em Nanotecnologia e Nanociências - NAP-NN (USP/SP)

17. Laboratório Central em Nanotecnologia - LCNano (UFPR/PR) 18. Laboratório de Fabricação e Caracterização de Nanodispositivos - Labdis (PUC/RJ).

Deve-se ressaltar que o tempo disponibilizado é contabilizado exclusivamente em projetos de nanotecnologia que é o foco e a razão de ser do SisNANO e que a contabilização do tempo de uso disponibilizado por cada laboratório terá também critérios qualitativos.

Para fazerem face a esses compromissos e desafios, os laboratórios do SisNANO receberão recursos de forma prioritária do MCTI. Já em 2013 o MCTI investirá R \$32 milhōes provenientes do Fundo Nacional de Desenvolvimento Científico e Tecnológico (FNDCT), $\mathrm{R} \$ 6,7$ milhôes provenientes do orçamento da CGNT e R \$25 milhōes do orçamento da Coordenação Geral de Serviços Tecnológicos (CGST) para formação de redes do Sistema Brasileiro de Tecnologia Sibratec com os laboratórios do SisNANO. 
O MCTI considera, ainda, essencial a participação das Fundações de Apoio à Pesquisa (FAPs) estaduais no SisNANO como agentes ativos, tanto na definição de diretrizes como no financiamento dos laboratórios nos estados de origem. A presença de um laboratório do SisNANO é, de fato, uma fator que modifica e enriquece o "ecossistema" de inovação em nanotecnologia em escala nacional e regional. Apoiar os laboratórios no nível estadual é, portanto, uma ação importante no sentido de impulsionar o desenvolvimento e a industrialização de nanotecnologias.

CONCLUSÃO A inovação só se concretiza quando o conhecimento técnico e científico é transformado em produtos e processos. A incorporação do progresso científico e tecnológico à produção industrial, gerando inovação, permanece como um grande desafio nacional. A transformação de nanotecnologias em produtos e bem-estar social é um processo complexo que passa também por questôes regulatórias que estão hoje postas como prioritárias em nível mundial. A Europa e os Estados Unidos, por exemplo, já atuam de forma articulada já que, sem regulação não há segurança jurídica (que inibe investimentos) e nem confiança por parte da sociedade de que a nanotecnologia é uma tecnologia que traz benefícios que estamos apenas começando a conhecer, desenvolver e utilizar.

A criação do CIN é um passo importante visto que a enorme tarefa de prover um ambiente profícuo à industrialização e comercialização de nanotecnologias só pode alcançado pela ação coordenada dos governos federal, estaduais e municipais.

Estamos certos que a estrutura do Programa Brasileiro de Nanotecnologia possui hoje os principais ingredientes para inserir o Brasil em uma nova onda de desenvolvimento econômico e social ancorada nas nanotecnologias.

Flávio Plentz é fisico, professor associado IV do Departamento de Física do Instituto de Ciências Exatas da Universidade Federal de Minas Gerais (UFMG) e atualmente é o coordenador geral de Micro e Nanotecnologias do Ministério da Ciência, Tecnologia e Inovação (MCTI).

Adalberto Fazzio é fisico e professor titular do Instituto de Física da Universidade de São Paulo (USP), membro na Academia Brasileira de Ciências $(A B C)$ e atualmente é secretário adjunto da Secretaria de Desenvolvimento Tecnológico e Inovação do MCTI.

\section{NOTAS E REFERÊNCIAS BIBLIOGRÁFICAS}

1. Knobel, M. “O futuro da nanotecnologia no Brasil. Vinte anos não são nada?", Cienc. Cult., vol.57, nº 1.2005.

2. Rossi-Bergmann, B."A nanotecnologia: da saúde para além do determinismo tecnológico", Cienc. Cult., vol.60, n² 2. 2008.

3. Site do programa "Desenvolvimento da Nanociência e Nanotecnologia" http://www.mct.gov.br/index.php/content/view/27136.html (Acesso em maio de 2013).

4. Site do Programa sobre Nanotecnologia: http://www.pdp.gov.br/ Paginas/detalhamento_programa.aspx?programa=Nanotecnolog ia\&path=Programas-Mobilizadores $\% 20$ em\%20\%C3\%A1reas\%20 Estrat\%C3\%A9gicas-Nanotecnologia (Acesso em maio de 2013).
5. Site do Fórum de Competitividade de Nanotecnologia: http://www. mdic.gov.br/sitio/interna/interna.php?area=3\&menu=2469 (Acesso em maio de 2013).

6. Site da Política de Desenvolvimento Produtivo (PDP): http://www. pdp.gov.br/ (Acesso em maio de 2013).

7. Site do $1^{\circ}$ e do $2^{\circ}$ Workshop http://workshopnano.abdi.com.br/default.aspx (Acesso em maio de 2013).

8. Ibidem

9. Site do periódico científico Environmental Toxicology and Chemistry http://onlinelibrary.wiley.com/doi/10.1002/etc.v31.1/issuetoc (Acesso em maio de 2013).

10. Alves, O. L.; Stéfani, D.; Parizotto, N. V. and Souza Filho, A. G. "Hydrotalcites: a highly efficient ecomaterial for effluent treatment originated from carbon nanotubes chemical processing". Journal of Physics: Conference Series, vol.304, 012024.2011.

11. Documento em PDF integral do General Safe Practices for Working with Engineered Nanomaterials in Research Laboratorie. http://www.cein.ucla.edu/PDFs/2012-147-Safe-Practices-NIOSH.pdf (Acesso em maio de 2013).

12. http://www.capes.gov.br/cooperacao-internacional/portugal/inl 